\title{
A Comparison of Mechanical Thrombectomy in the M1 and M2 Segments of the Middle Cerebral Artery: A Review of 585 Consecutive Patients
}

\author{
Pervinder Bhogal ${ }^{a} \quad$ Philipp Bücke ${ }^{b} \quad$ Muhammad AlMatter $^{a}$ \\ Oliver Ganslandt ${ }^{c}$ Hansjörg Bäzner ${ }^{b}$ Hans Henkes ${ }^{a}{ }^{d}$ \\ Marta Aguilar Pérez ${ }^{a}$ \\ ${ }^{a}$ Neuroradiological Clinic, ${ }^{b}$ Neurological Clinic, and ${ }^{\mathrm{c} N e u r o s u r g i c a l ~ C l i n i c, ~ N e u r o c e n t e r, ~}$ \\ Klinikum Stuttgart, Stuttgart, and dMedical Faculty, University Duisburg-Essen, Duisburg, \\ Germany
}

\section{Keywords}

Stroke $\cdot$ Mechanical thrombectomy $\cdot M 1$ segment $\cdot M 2$ segment

\begin{abstract}
Background: Mechanical thrombectomy for anterior-circulation large-vessel occlusion has shown benefit; however, the question of whether this technique is safe and effective in the distal vasculature remains unanswered. We sought to compare the outcome data from mechanical thrombectomy of the M2 branches of the middle cerebral artery (MCA) with those of the M1 segment. Methods: We performed a retrospective analysis of prospectively collected data of patients with acute ischaemic stroke undergoing mechanical thrombectomy of isolated M1 or M2 branches of the MCA between August 2008 and August 2016. Results: We identified 585 patients, 479 with M1 occlusions and 106 with M2 occlusions. The average age was $72 \pm$ 12.8 and $68 \pm 13.8$ years, respectively $(p=0.007)$. The baseline Alberta Stroke Program Early Computed Tomographic (ASPECT) score was similar in both cohorts, but patients with M1 occlusions presented with higher mean National Institutes of Health Stroke Scale (NIHSS) scores of 15.7 compared to $11.8(p<0.001)$. There was no significant difference in the average procedure time for each cohort; fewer thrombectomy attempts were required in the M2 cohort (2.3 vs. $1.8, p=0.0004$ ), but the overall time to recanalization was longer in the M2 cohort (353 vs. $399 \mathrm{~min}, p<0.001$ ). Similar rates of successful reperfusion (Thrombolysis in Ischaemic Stroke score $[\mathrm{TICI}] \geq 2 \mathrm{~b} 88.5$ vs. $90.5 \%, p=0.612$ ) were seen, but food outcome (modified Rankin Scale $\leq 2$ ) was lower in M1 occlusions (37.2 vs. $54.3 \%, p<0.001$ ). Rates of symptomatic intracranial haemorrhage were similar. Conclusion: Good clinical outcomes can be achieved for both groups with no significant differences in procedure length, final TICI recanalization rates or intracranial haemorrhage between the M1 and M2 cohorts. ( 2017 The Author(s)




\section{Introduction}

Conclusive evidence regarding the significant benefit of mechanical thrombectomy compared to intravenous thrombolysis was published in a series of trials during 2015. However, despite the overwhelming data obtained from these trials, there are still unanswered questions. One of the most frequently asked questions is whether the results of these trials can be extrapolated to the more distal vasculature of the anterior circulation. The inclusion criteria varied between the different trials with Multicentre Randomized Clinical Trial of Endovascular Treatment for Acute Ischaemic Stroke in the Netherlands (MR CLEAN) [1] and Extending the Time for Thrombolysis in Emergency Neurological Deficits - IntraArterial (EXTEND IA) [2] allowing the inclusion of patients with occlusions of the M2 segment of the middle cerebral artery (MCA). However, despite this, only 22 patients with solitary M2 occlusions were enrolled in these 2 trials. Similarly, whilst Endovascular Treatment for Small Core and Anterior Circulation Proximal Occlusion with Emphasis on Minimizing CT to Recanalization Times (ESCAPE) [3], Randomized Trial of Revascularisation with Solitaire FR Device versus Best Medical Therapy in the Treatment of Acute Stroke due to Anterior Circulation Stroke within Eight Hours of Symptom Onset (REVASCAT) [4] and Solitaire with the Intention of Thrombectomy as Primary Endovascular Treatment (SWIFT PRIME) [5] prohibited the inclusion of patients with M2 occlusions, a review by the core laboratories identified a number of patients that were initially wrongly included. In total, across the 5 major trials, 51 patients with M2 occlusions were enrolled compared to $439 \mathrm{M} 1$ occlusions in total across the 5 trials, and as yet, there is no firm guidance on whether M2 occlusions should be treated with mechanical thrombectomy.

We report the outcome of patients with acute occlusions of M2 vessels in comparison to occlusions of the M1 segment of the MCA after intra-arterial mechanical thrombectomy with second-generation devices. We also sought to evaluate the safety of these devices with respect to the M1 segment.

\section{Patients and Methods}

\section{Study Design and Patients}

This is a single-centre retrospective case series analysis of consecutive patients with either M1 or M2 branch occlusions who underwent intra-arterial mechanical thrombectomy at our institution between August 1, 2008, and August 1, 2016. Our hospital runs a comprehensive stroke centre with neurological, neurosurgical and neuroradiological services and serves as both a primary and secondary referral centre for acute intravenous treatment and/or intra-arterial treatment for stroke. We serve an immediate population of over 500,000 individuals and several million individuals in the greater metropolitan area. Data were available from our local prospectively maintained database.

After clinical assessment, anterior circulation occlusion was confirmed on either computed tomography (CT) plus CT angiography or magnetic resonance imaging (MRI), which included MR angiography, fluidattenuated inversion recovery (FLAIR), diffusion-weighted imaging (DWI), and T2 gradient recalled echo (GRE) or susceptibility-weighted imaging (SWI) sequences. The decision between MRI and CT was made individually and was based on the patient's condition and MRI eligibility and availability. Intra-arterial treatment with mechanical thrombectomy was based on the judgement of the operator and attending neurologist/stroke physician. Intravenous recombinant tissue plasminogen activator was administered in accordance with the appropriate management guidelines.

Treatment of occluded anterior circulation vessels was based on the judgement of the admitting stroke neurologist at our centre or at the referring centre and of the interventional neuroradiologist. Our local protocol does not exclude patients based on time thresholds, and we treat patients with an unknown stroke onset if there is evidence of potentially salvageable brain tissue. An upper age limit for performing mechanical thrombectomy is not used at our institution; rather, the pre-morbid status of the patient is taken into consideration along with co-morbidities and the will of the patient or family members. 
In our analysis, we included all patients in whom the clot could be positively identified as starting in either the M1 or a single M2 branch, and complete imaging was available on PACS for review. We excluded patients with incomplete imaging.

\section{Demographics, Variables and Outcome Measures}

Past medical history of hypertension, atrial fibrillation, diabetes mellitus and smoking was recorded, as were standard demographics of age and gender. Neurological characteristics of the patients as assessed by means of the National Institutes of Health Stroke Scale (NIHSS) prior to treatment were also recorded and retrieved from our database.

\section{Imaging Analysis}

Early ischaemic change was measured using the Alberta Stroke Program Early Computed Tomographic (ASPECT) score on non-contrast-enhanced axial CT head scans. For patients with MRI, the DWI sequence was used to determine the ASPECT score. The Thrombolysis in Ischaemic Stroke score (TICI) was used to determine recanalization with successful reperfusion (partial and complete) defined as TICI $\geq 2 \mathrm{~b}$ (range: 0 , no reperfusion; 3 , full reperfusion with filling of all distal branches). At the end of the procedure, the operator assigned the final TICI score. Post-operative imaging was performed between 24 and $36 \mathrm{~h}$ using either CT or MRI with the post-operative ASPECT score recorded in the database.

\section{Study Outcomes}

Clinical outcome was measured with the modified Rankin Scale (mRS) at 90 days after stroke (range 0-6 with lower scores indicating better outcomes). A good outcome was classified as $\mathrm{mRS} \leq 2$ and a poor outcome as $\mathrm{mRS} \geq 3$. Other secondary outcomes included the presence of haemorrhage on post-operative imaging, recorded according to the method used in the European Australasian Acute Stroke Study (ECASS) trials: parenchymal haematoma (PH-1, PH-2) and haemorrhagic infarction (HI-1, HI-2) [6]. Procedure length (defined as the groin puncture time until the final angiographic run) and time to recanalization were also retrieved from our database.

\section{Statistical Analysis}

The Student $t$ test was used to evaluate all metric variables, and the Fisher exact test was used to evaluate all other variables. All $p$ values are 2 sided, and $p<0.05$ was considered significant. Calculations were performed using Stata/IC 14.2 for Windows (StataCorp LP, College Station, TX, USA).

\section{Results}

A total of 619 patients were screened (501 patients with M1 occlusions and 118 with M2 occlusions). A total of 585 patients (294 male, 50.2\%) were included in our analysis with the remaining 34 patients excluded because of incomplete imaging. There were 479 patients in the M1 cohort with a mean age of $72 \pm 12.8$ years. There were 106 patients in the M2 cohort with a mean age of $68 \pm 13.8$ years. Patients in the M2 cohort were significantly younger ( $p=$ 0.007). Associated medical conditions were common in both groups with hypertension being the most prevalent associated condition ( 73.9 vs. $70.7 \%, p=0.54$ ) and atrial fibrillation also seen in over half of the patients in both groups ( $56.9 \mathrm{vs.} 50.9 \%, p=0.28)$. The degree of early ischaemic changes visible on either non-enhanced axial CT scans or DWI MRI was similar with a mean average ASPECT score of 8.5 for both M1 and M2 occlusions (scores ranging from 0 to 10 with higher scores indicating less areas of damaged brain). Patients with M1 occlusions presented with a higher mean NIHSS score (27.1 vs. $19.8 \%, p=0.14$, for moderately severe and 37.2 vs. $8.49 \%, p=0.003$, for severe strokes). The results are summarised in Table 1.

Mechanical thrombectomy was performed using stent-retrievers in 475 patients (99.2\%) with M1 occlusions and using aspiration alone in 4 patients. Stent-retrievers were used in all cases of M2 occlusions. The average time taken to perform the procedure was similar. The time from stroke onset to recanalization was shorter in the M1 cohort (353 vs. 399 min, $p<$ 
Bhogal et al.: A Comparison of Mechanical Thrombectomy in the M1 and M2 Segments of the Middle Cerebral Artery: A Review of 585 Consecutive Patients

Table 1. Baseline characteristics of patients in both cohorts

\begin{tabular}{|c|c|c|c|}
\hline Characteristics & $\begin{array}{l}\text { M1 cohort } \\
(n=479)\end{array}$ & $\begin{array}{l}\text { M2 cohort } \\
(n=106)\end{array}$ & $p$ value \\
\hline Mean age $\pm S D$, years & $72 \pm 12.8$ & $68 \pm 13.8$ & 0.007 \\
\hline Male & $236(49.3)$ & $58(54.7)$ & 0.335 \\
\hline \multicolumn{4}{|l|}{ Associated conditions } \\
\hline Atrial fibrillation & $273(56.9)$ & $54(50.9)$ & 0.28 \\
\hline Diabetes mellitus & 109 (22.7) & $31(29.2)$ & 0.167 \\
\hline Hypertension & 354 (73.9) & $75(70.7)$ & 0.544 \\
\hline Hypercholesterolaemia & $138(28.8)$ & $29(27.3)$ & 0.813 \\
\hline Smoker & $64(13.6)$ & $21(19.8)$ & 0.095 \\
\hline \multicolumn{3}{|l|}{ Suspected cause } & 0.416 \\
\hline Cardiac embolism & $248(51.8)$ & $46(43.4)$ & \\
\hline Extra-cranial atherosclerosis & 91 (18.9) & $22(20.8)$ & \\
\hline Dissection & $11(2.3)$ & $3(2.8)$ & \\
\hline Unknown & $129(26.9)$ & $35(33)$ & \\
\hline \multicolumn{3}{|l|}{ NIHSS score on admission $^{\mathrm{a}}$} & $<0.001$ \\
\hline 0 & $2(0.4)$ & $1(0.9)$ & \\
\hline $1-4$ & $35(7.5)$ & $17(16)$ & \\
\hline $5-15$ & $207(44.5)$ & $58(54.7)$ & \\
\hline $16-20$ & $126(27.1)$ & $21(19.8)$ & \\
\hline $21-42$ & $95(20.4)$ & $9(8.49)$ & \\
\hline Pre-operative MRI & $154(32.2)$ & $47(44.3)$ & \\
\hline Pre-operative CT & $324(67.8)$ & $59(55.7)$ & \\
\hline Pre-operative ASPECT score & $8.5 \pm 1.88$ & $8.5 \pm 1.7$ & 1 \\
\hline \multicolumn{3}{|l|}{ Side } & 0.914 \\
\hline Left & $222(46.3)$ & $50(47.1)$ & \\
\hline Right & $257(53.7)$ & $56(52.9)$ & \\
\hline Mean duration of procedure $\pm \mathrm{SD}$, min & $103 \pm 2.8$ & $103 \pm 3.4$ & 1 \\
\hline \multirow{2}{*}{$\begin{array}{l}\text { Mean number of thrombectomy attempts } \pm \text { SD } \\
\text { Range }\end{array}$} & $2.3 \pm 1.7$ & $1.8 \pm 1.2$ & 0.0004 \\
\hline & $1-11$ & $1-8$ & \\
\hline \multirow{2}{*}{$\begin{array}{l}\text { Mean time from stroke onset to recanaliza- } \\
\text { tion } \pm \mathrm{SD}, \min ^{\mathrm{b}}\end{array}$} & & & $<0.001$ \\
\hline & $353 \pm 5.1$ & $399 \pm 7.5$ & \\
\hline
\end{tabular}

Figures are numbers with percentages in parentheses unless otherwise indicated. Italics indicate statistical significance. SD, standard deviation; NIHSS, National Institutes of Health Stroke Scale; MRI, magnetic resonance imaging; CT, computed tomography; ASPECT score, Alberta Stroke Program Early Computed Tomographic score. ${ }^{\mathrm{a}}$ M1 cohort: $n=465$; M2 cohort: $n=106 .{ }^{\mathrm{b}}$ M1 cohort: $n=388$; M2 cohort: $n=82$.

0.001) despite overall similar procedure length times. This may reflect a delay in the patients, clinicians or both recognising the symptoms of stroke in case of more distal occlusions. Similarly, the mean number of thrombectomy attempts was also significantly lower in the M2 cohort (2.3 vs. $1.8, p=0.0004)$.

The average post-operative ASPECT score was significantly lower for patients with M1 occlusions (6.2 vs. 7.2, $p<0.001$ ) despite similar rates of successful reperfusion (TICI $\geq 2 \mathrm{~b}$ 88.5 vs. $90.5 \%, p=0.612$ ). A clinically good outcome (mRS $\leq 2$ ) was seen in fewer patients with M1 occlusions ( 37.2 vs. $54.3 \%, p<0.001$ ) with higher rates of poor outcome in the M1 cohort ( 62.8 vs. $45.3 \%, p<0.001$ ) and higher mortality rates overall in the M1 cohort $(24.4$ vs. 14.2\%) (Fig. 1; Table 2). The rates of symptomatic intracranial haemorrhage (SICH) were similar in the 2 groups and similar to those seen in the major trials [7]. The post-operative results are summarised in Table 2 .

We performed a multivariate logistic regression to predict the influence of age, NIHSS score, pre-operative ASPECT score and clot location on the probability of a good outcome 


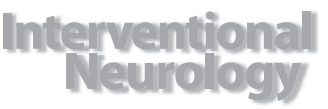

Table 2. The post-operative outcome of both cohorts
Fig. 1. Comparison of outcomes at 90 days between the M1 and M2 cohorts.

\begin{tabular}{l|l}
\hline Intervent Neurol 2017;6:191-198 \\
\hline DOI: 10.1159/000475535 & $\begin{array}{l}\text { @ 2017 S. Karger AG, Basel } \\
\text { www.karger.com/ine }\end{array}$ \\
\hline
\end{tabular}

Bhogal et al.: A Comparison of Mechanical Thrombectomy in the M1 and M2 Segments of the Middle Cerebral Artery: A Review of 585 Consecutive Patients

\begin{tabular}{lccc}
\hline Characteristics & $\begin{array}{l}\text { M1 cohort } \\
(n=470)\end{array}$ & $\begin{array}{l}\text { M2 cohort } \\
(n=106)\end{array}$ & $p$ value \\
\hline Mean post-operative & & & \\
ASPECTS score \pm SD & $6.2 \pm 2.7$ & $7.2 \pm 1.7$ & $<0.001$ \\
Post-operative haemorrhage & & 0.575 \\
None & $371(77.5)$ & $78(73.6)$ & \\
SAH & $27(5.6)$ & $6(5.7)$ & \\
HI-1 & $12(2.5)$ & $6(5.7)$ & \\
HI-2 & $21(4.4)$ & $6(5.7)$ & \\
PH-1 & $24(5)$ & $6(5.7)$ & \\
PH-2 & $24(5)$ & $4(3.8)$ & \\
SICH & $25(5.2)$ & $5(4.71)$ & 1 \\
TICI $\geq 2 b$ & $424(88.5)$ & $96(90.5)$ & 0.612 \\
TICI 3 & $287(59.9)$ & $73(68.9)$ & 0.098 \\
\hline 90 -day mRS & & & \\
0 & $65(13.6)$ & $25(23.5)$ & \\
1 & $63(13.2)$ & $23(21.7)$ & \\
2 & $50(10.4)$ & $10(9.4)$ & \\
3 & $88(18.4)$ & $18(16.9)$ & \\
4 & $64(13.3)$ & $8(7.5)$ & \\
5 & $32(6.7)$ & $7(6.6)$ & \\
6 & $117(24.4)$ & $15(14.2)$ & \\
$\leq 2$ & $178(37.2)$ & $58(54.7)$ & 0.001 \\
$3-6$ & $301(62.8)$ & $48(45.3)$ & 0.001 \\
\hline
\end{tabular}

Figures are numbers with percentages in parentheses unless otherwise indicated. Italics indicate statistical significance. ASPECT score, Alberta Stroke Program Early Computed Tomographic score; SD, standard deviation; SAH, subarachnoid haemorrhage; HI, haemorrhagic infarction; $\mathrm{PH}$, parenchymal haematoma; SICH, symptomatic intracranial haemorrhage; TICI, Thrombolysis in Ischaemic Stroke score; mRS, modified Rankin Scale.

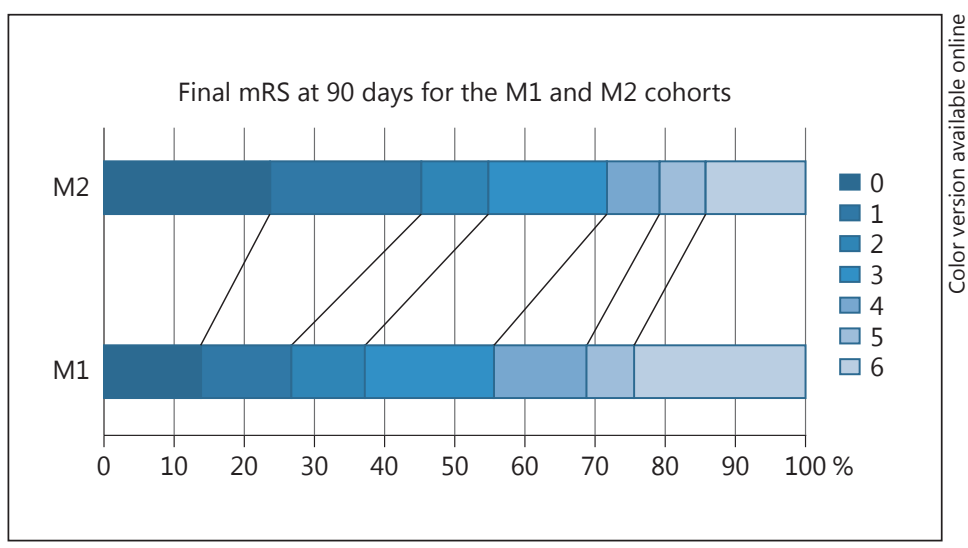

(mRS $\leq 2)$ and on the probability of SICH. The probability of a good outcome was significantly influenced by age ( $p<0.001$, OR $0.96,95 \%$ CI $0.94-0.97)$ and NIHSS score $(p<0.001$, OR $0.9,95 \%$ CI $0.87-0.93$ ). The older a patient or the higher the NIHSS score, the smaller was the chance for a good outcome. The pre-operative ASPECT score did not influence the chance of a good outcome ( $p=0.241)$, nor did clot location ( $p=0.261)$. Only the NIHSS score 
was significantly associated with SICH ( $p=0.013$, OR 1.06, 95\% CI 1.01-1.12). Age, preoperative ASPECT score and clot location did not influence the probability of SICH significantly.

\section{Discussion}

The endovascular treatment of large-vessel occlusions in the anterior circulation has been shown to be an extremely effective treatment strategy with excellent rates of recanalization, low rates of complications and improved patient outcomes compared to best medical management [1-5]. However, given that these studies enrolled only 51 patients with M2 occlusions into the endovascular treatment arm, representing only $4 \%$ of the total number of patients enrolled, extrapolation of the effectiveness of mechanical thrombectomy to these smaller vessels has been difficult. Despite these challenges, there is evidence that the M2 branches are amenable to thrombectomy and that good results can be obtained. Sarraj et al. [8] recently published their results from a retrospective cohort study of pooled patients with acute ischaemic strokes with involvement of isolated M2 vessels from 10 US centres. This study identified 522 patients, of whom 288 underwent mechanical thrombectomy and 234 received best medical management. In this study, the rate of a good outcome was higher for those undergoing endovascular treatment $(62.8 \%$ with $\mathrm{mRS} \leq 2)$ than for those with medical management ( $35.4 \%$ with $\mathrm{mRS} \leq 2)$. Even after adjustment for age and NIHSS score, the endovascular treatment cohort had 3 times the odds of a good outcome compared to those with best medical management with no statistically significant difference between the 2 groups for SICH. Other studies have looked at the safety and outcomes of patients undergoing mechanical thrombectomy of M2 vessels in comparison to M1 vessels. Dorn et al. [9] published a comparative study that involved 104 M1 occlusions and 15 M2 occlusions. They showed that a good clinical outcome was more frequent in the M2 group than in the M1 group (60 vs. $43.3 \%$ for $\mathrm{mRS} \leq 2$ ) and that mortality was higher in the M1 group (21.2 vs. $6.7 \%$ ). In our cohort, we also observed a good outcome for patients with M2 occlusions (54.6 vs. $37.2 \%$ for $\mathrm{mRS} \leq 2$ ) as well as a higher rate of mortality in the M1 cohort (24.4 vs. 14.2\%). A further study by Coutinho et al. [10] looked at the outcomes of patients enrolled into the STAR, SWIFT and SWIFT PRIME studies with M2 occlusions. In their analysis of 50 patients with M2 occlusions and 249 patients with M1 occlusions, they showed non-significant differences in successful reperfusion (TICI $\geq 2 \mathrm{~b}$ reperfusion) or in good clinical outcomes at follow-up in the 2 groups ( $60 \%$ for M2 occlusions and 54\% for M1 occlusions). Similarly, they found no significant differences in SICH or mortality. In our cohort, there was no significant difference in successful reperfusion; however, we observed a significantly better outcome for patients with M2 occlusions despite a longer time to recanalization.

Given the more distal location of M2 occlusions, the smaller calibre of the vessels and the reduction in affected brain volume associated with more distal obstructions, it has been suggested that the risk of thrombectomy may not be warranted. However, according to the data of Dorn et al. [9], Coutinho et al. [10] and Sarraj et al. [8] as well as our own data, this does not appear to be the case. Furthermore, the idea that the more distal location of the occlusion and the potentially smaller brain volume affected should deter physicians from pursuing mechanical recanalization is also disputable, since in the study of Sheth et al. [11] one-third of patients with M2 occlusions had infarct volumes of $\geq 50 \mathrm{~mL}$ and $11 \%$ had infarct volumes of $\geq 90 \mathrm{~mL}$ [11]. Therefore, from available evidence it appears that mechanical thrombectomy of the M2 segment is as safe as thrombectomy of the M1 segment and that, in both scenarios, there can be a large volume of potentially salvageable brain tissue. The more distal location does not appear to affect the recanalization rate or procedure length with TICI 
$\geq 2 \mathrm{~b}$ achieved in $88.5 \%$ of $\mathrm{M} 1$ occlusions and $90.5 \%$ of patients in our study and virtually identical procedural times. In our cohort, significantly fewer thrombectomy attempts were required to restore recanalization, and this may be due to smaller clots in the M2 branches, different stent-clot interactions or some other unidentified factors. Overall, the similar preoperative ASPECT score and the better post-operative ASPECT score in the M2 cohort, in unison with longer overall recanalization times and similar operative times compared to the M1 cohort, would suggest that the ability of the collateral supply to meet the demands of the hypoxic brain is better in M2 occlusions.

This then leads us to the appropriate selection of patients with M2 occlusions. The asymmetry of the MCA branches can have an impact on both the volume of at-risk brain as well as on the NIHSS score, which is biased towards the anterior division of the MCA, particularly in the dominant hemisphere [12] where the receptive language centres are based. Although we did not detect a significant difference in the laterality of the strokes, we observed a significantly longer time to recanalization despite overall procedure lengths being similar. This may be related to a delay in the patient, physician or both suspecting stroke and, hence, a delay in instituting the appropriate management. In our cohort, patients in the M2 cohort presented with lower NIHSS scores (mean 11.8 vs. $15.7, p<0.001$ ) with a significantly larger number of patients in the M2 cohort presenting with low NIHSS scores of $1-4$ ( 7.5 vs. $16 \%, p=0.009)$. Despite the inherent problem with the NIHSS score, we feel that a low NIHSS score should not exclude patients from mechanical thrombectomy, particularly as it has recently been shown for M1 occlusions that mechanical thrombectomy improved outcomes in patients with low NIHSS scores [13]. Although we currently do not use CT or MR perfusion in our decisionmaking process, we feel that this may provide the most useful information on potentially salvageable brain tissue as well as allowing the potential assessment of which functional territories can be saved. This strategy of penumbral imaging was successfully used in the SWIFT PRIME and EXTEND IA trials, and it may at least partially explain the better outcomes seen in these 2 trials compared to the other major trials ( 60 and $72 \% \mathrm{mRS} \leq 2$ for SWIFT PRIME and EXTEND IA, respectively).

The intracranial haemorrhage rate in our cohort was higher than reported in the major thrombectomy trials, and we are unsure as to the exact reason for this. It is possible that the broader inclusion criteria applied to our patients compared to those of the main trials may have had an effect on the haemorrhage rate. The multivariate regression analysis showed that only the NIHSS score was statistically significantly associated with SICH, and although there did not appear to be a major difference in the NIHSS scores of our patients compared to the major trials, this cannot be completely excluded.

Our study has several limitations. Despite the inherent limitations of a retrospective design, the baseline characteristics, besides age, were similar in the 2 groups. A greater proportion of patients in the M1 cohort had severe stroke at admission, which is likely due to the larger brain territory being affected; however, this may have skewed the M1 results to be more negative.

\section{Conclusion}

Despite the inherent limitations of a retrospective design, our data come from the largest single-centre study comparing the outcomes between M1 and M2 occlusions. Our data suggest that M2 thrombectomy is as safe as M1 thrombectomy and that a significant proportion of patients achieve a good clinical outcome. 


\begin{tabular}{l|l}
\hline DOI: $10.1159 / 000475535$ & $\begin{array}{l}\text { C } 2017 \text { S. Karger AG, Basel } \\
\text { www.karger.com/ine }\end{array}$ \\
\hline
\end{tabular}

Bhogal et al.: A Comparison of Mechanical Thrombectomy in the M1 and M2

Segments of the Middle Cerebral Artery: A Review of 585 Consecutive Patients

\section{Acknowledgements}

We would like to thank Hiltrud Niggemann for her assistance with the statistical analysis.

\section{Statement of Ethics}

The local ethics committee approved data collection and analysis. The patient or legal representative provided written informed consent for data entry into the database, data analysis, and anonymised data publication. The study is in accordance with the ethical standards laid down in the 1964 Declaration of Helsinki and later amendments.

\section{Disclosure Statement}

M. Aguilar Pérez and P. Bhogal serve as proctors and consultants for phenox GmbH with moderate financial compensation. H. Henkes is a co-founder and shareholder of phenox GmbH. The other authors have no potential conflicts of interest.

\section{Funding Sources}

This research received no specific grant from any funding agency in the public, commercial or not-forprofit sectors.

\section{References}

1 Berkhemer OA, Fransen PSS, Beumer D, van den Berg LA, Lingsma HF, Yoo AJ, et al: A randomized trial of intraarterial treatment for acute ischemic stroke. N Engl J Med 2015;372:11-20.

2 Campbell BCV, Mitchell PJ, Kleinig TJ, Dewey HM, Churilov L, Yassi N, et al: Endovascular therapy for ischemic stroke with perfusion-imaging selection. N Engl J Med 2015;372:1009-1018.

3 Goyal M, Demchuk AM, Menon BK, Eesa M, Rempel JL, Thornton J, et al: Randomized assessment of rapid endovascular treatment of ischemic stroke. N Engl J Med 2015;372:1019-1030.

4 Jovin TG, Chamorro A, Cobo E, de Miquel MA, Molina CA, Rovira A, et al: Thrombectomy within $8 \mathrm{~h}$ after symptom onset in ischemic stroke. N Engl J Med 2015;372:2296-2306.

5 Saver JL, Goyal M, Bonafe A, Diener H-C, Levy EI, Pereira VM, et al: Stent-retriever thrombectomy after intravenous t-PA vs. t-PA alone in stroke. N Engl J Med 2015;372:2285-2295.

6 Berger C, Fiorelli M, Steiner T, Schäbitz WR, Bozzao L, Bluhmki E, et al: Hemorrhagic transformation of ischemic brain tissue: asymptomatic or symptomatic? Stroke J Cereb Circ 2001;32:1330-1335.

7 Goyal M, Menon BK, van Zwam WH, Dippel DWJ, Mitchell PJ, Demchuk AM, et al: Endovascular thrombectomy after large-vessel ischaemic stroke: a meta-analysis of individual patient data from five randomised trials. Lancet 2016;387:1723-1731.

8 Sarraj A, Sangha N, Hussain MS, Wisco D, Vora N, Elijovich L, et al: Endovascular therapy for acute ischemic stroke with occlusion of the middle cerebral artery M2 segment. JAMA Neurol 2016;73:1291-1296.

9 Dorn F, Lockau H, Stetefeld H, Kabbasch C, Kraus B, Dohmen C, et al: Mechanical thrombectomy of M2-occlusion. J Stroke Cerebrovasc Dis 2015;24:1465-1470.

10 Coutinho JM, Liebeskind DS, Slater L-A, Nogueira RG, Baxter BW, Levy EI, et al: Mechanical thrombectomy for isolated M2 occlusions: a post hoc analysis of the STAR, SWIFT, and SWIFT PRIME studies. AJNR Am J Neuroradiol 2016;37:667-672.

11 Sheth SA, Yoo B, Saver JL, Starkman S, Ali LK, Kim D, et al: M2 occlusions as targets for endovascular therapy: comprehensive analysis of diffusion/perfusion MRI, angiography, and clinical outcomes. J Neurointerv Surg 2015; 7:478-483.

12 Fink JN, Selim MH, Kumar S, Silver B, Linfante I, Caplan LR, et al: Is the association of National Institutes of Health Stroke Scale scores and acute magnetic resonance imaging stroke volume equal for patients with rightand left-hemisphere ischemic stroke? Stroke 2002;33:954-958.

13 Haussen DC, Bouslama M, Grossberg JA, Anderson A, Belagage S, Frankel M, et al: Too good to intervene? Thrombectomy for large vessel occlusion strokes with minimal symptoms: an intention-to-treat analysis. J Neurointerv Surg 2016, Epub ahead of print. 